\title{
Ability of children to perform touchscreen gestures and follow prompting techniques when using mobile apps
}

\author{
Savita Yadav, MTech, Pinaki Chakraborty, PhD, Arshia Kaul, BE, Pooja, BE, Bhavya Gupta, BE, Anchal Garg, BE
}

Department of Computer Science and Engineering, Netaji Subhas University of Technology, New Delhi, India

Background: Children today get access to smartphones at an early age. However, their ability to use mobile apps has not yet been studied in detail.

Purpose: This study aimed to assess the ability of children aged 2-8 years to perform touchscreen gestures and follow prompting techniques, i.e., ways apps provide instructions on how to use them.

Methods: We developed one mobile app to test the ability of children to perform various touchscreen gestures and another mobile app to test their ability to follow various prompting techniques. We used these apps in this study of 90 children in a kindergarten and a primary school in New Delhi in July 2019. We noted the touchscreen gestures that the children could perform and the most sophisticated prompting technique that they could follow.

Results: Two- and 3-year-old children could not follow any prompting technique and only a minority $(27 \%)$ could tap the touchscreen at an intended place. Four- to 6-year-old children could perform simple gestures like a tap and slide (57\%) and follow instructions provided through animation (63\%). Sevenand 8-year-old children could perform more sophisticated gestures like dragging and dropping (30\%) and follow instructions provided in audio and video formats (34\%). We observed a significant difference between the number of touchscreen gestures that the children could perform and the number of prompting techniques that they could follow $(F=544.0407$, $P<0.05)$. No significant difference was observed in the performance of female versus male children $(P>0.05)$.

Conclusion: Children gradually learn to use mobile apps beginning at 2 years of age. They become comfortable performing single-finger gestures and following nontextual prompting techniques by 8 years of age. We recommend that these results be considered in the development of mobile apps for children.

Key words: Child, Smartphone, Touchscreen gesture, Prompting technique

\section{Key message}

Question: At what age children develop skills necessary for using mobile apps?

Finding: Four- to 6-year-old children can perform single-finger touchscreen gestures and follow nontextual prompting techniques, while some 7- and 8-year-old children can also perform multifinger gestures and follow textual instructions.

Meaning: Mobile apps for young children should make minimal use of text and only single-finger touchscreen gestures should be required to operate them.

\section{Introduction}

Children are attracted to smartphones more than other household gadgets ${ }^{1)}$ and such a new form of media can affect their wellbeing. ${ }^{2)}$ Smartphones invoke curiosity and interest among children. By the age of 2 years, children can hold a smartphone firmly in their hands and scribble and draw using a simple draw. ing app. ${ }^{3)}$ Children explore different types of mobile apps as they grow up. ${ }^{4)}$ Mobile apps with age appropriate and engaging content and an interactive interface can be used to foster informal learning among young children., ${ }^{5,6}$ However, $\mathrm{Chau}^{7}$ found that many mobile apps marketed for children are not actually suitable for them. Mobile apps for children should have a user interface that takes into consideration the physical and cognitive capabilities of children, ${ }^{8)}$ and use appropriate ways for providing instructions to children on how to use them. ${ }^{9}$ Children acquire skills necessary for using mobile apps gradually as they grow up. If the capabilities of children belonging to different age groups are known to software developers, then they can develop suitable mobile apps for them. Ability to perform touchscreen gestures and follow in-app instructions are 2 important skills necessary for using mobile apps. In this study, we evaluate these 2 skills of children.

Users interact differently with personal computers and smartphones even when they are using the same software, and their interaction with smartphones is strongly influenced by their ability to perform various touchscreen gestures. Preliminary

Corresponding author: Pinaki Chakraborty, PhD. Department of Computer Science and Engineering, Netaji Subhas University of Technology, New Delhi 110078, India 凶E-mail: pinaki_chakraborty_163@yahoo.com, https://orcid.org/0000-0002-2010-8022

Received: 18 August, 2019, Revised: 8 January, 2020, Accepted: 10 January, 2020

This is an open-access article distributed under the terms of the Creative Commons Attribution Non-Commercial License (http://creativecommons.org/licenses/bync/4.0/) which permits unrestricted non-commercial use, distribution, and reproduction in any medium, provided the original work is properly cited. 


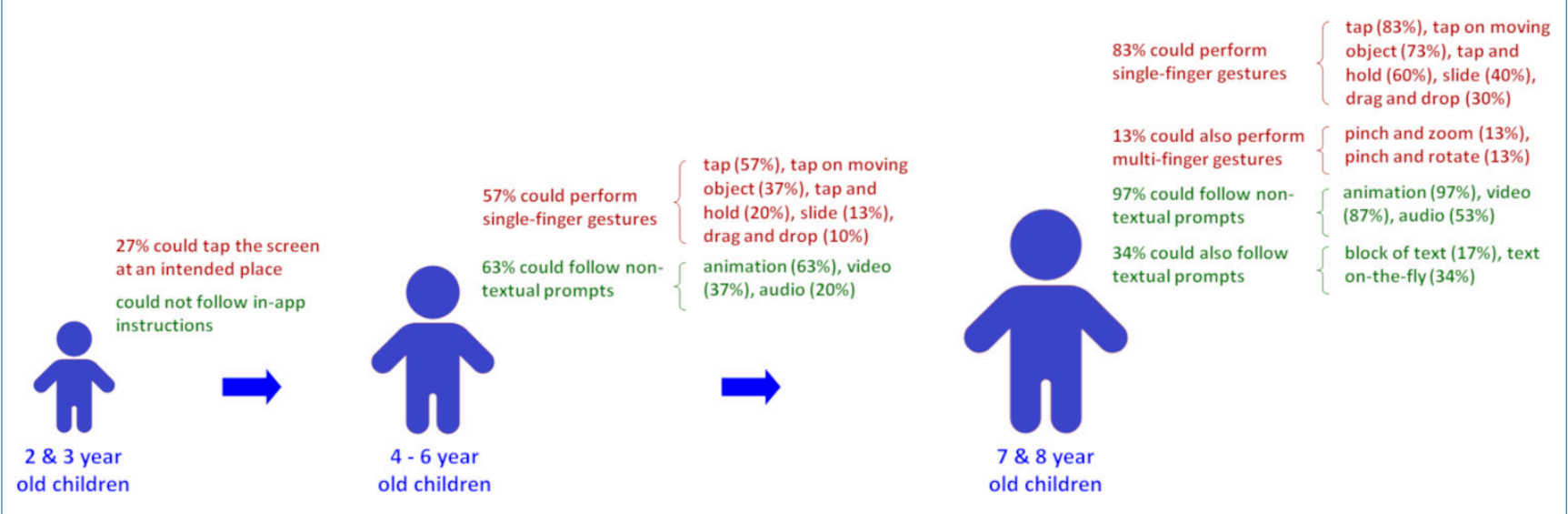

Graphical abstract. Difference between number of gestures performed by children and number of prompting techniques they could follow is significant $(P<0.05)$. Difference in performance of female and male children is insignificant $(P>0.05)$.

studies found that 2- and 3-year-old children can perform gestures like tap, slide and flick, ${ }^{10)}$ while 4- to 6-year-old children can perform more complex gestures like drag and drop and pinch and zoom. ${ }^{10,11)}$ Nacher et al. ${ }^{12)}$ experimented with interface designs that allow children to perform touchscreen gestures with more ease.

Mobile apps typically use text, audio and animation to provide instructions to users on how to use them. Young children cannot follow in-app prompting techniques used by mobile apps and need an adult to guide them when using mobile apps. Hiniker et al. ${ }^{13)}$ observed that children start following the instructions provided by mobile apps around the age of 5 years and can then operate a smartphone alone. McKnight and Fitton ${ }^{14)}$ observed that children often have problem in understanding the language of the instructions provided by mobile apps.

The objective of our study was to understand the ability of 2- to 8-year-old children to perform touchscreen gestures and follow in-app prompting techniques.

\section{Methods}

\section{The apps}

We developed a mobile app named Baby's Touch to assess the ability of children to perform various touchscreen gestures (Fig. 1A). The app displays 4 simple geometric figures, viz. a circle, a square, a triangle and a star, on a simple background. A child is asked to perform a particular touchscreen gesture on all the 4 geometric objects. The time taken by the child to do so is noted. If the child can perform the touchscreen gesture on all the 4 geometric objects within a predefined time limit, then we say that the child is able to perform that touchscreen gesture successfully. We say that the child has failed to perform that touchscreen gesture, otherwise. The app can identify 7 gestures, viz. tap on a stationary object, tap on a moving object, tap and hold, slide, drag and drop, pinch and zoom, and pinch and rotate.

We developed another mobile app, named Toddler's Tiles, to assess the ability of children to follow various in-app prompting

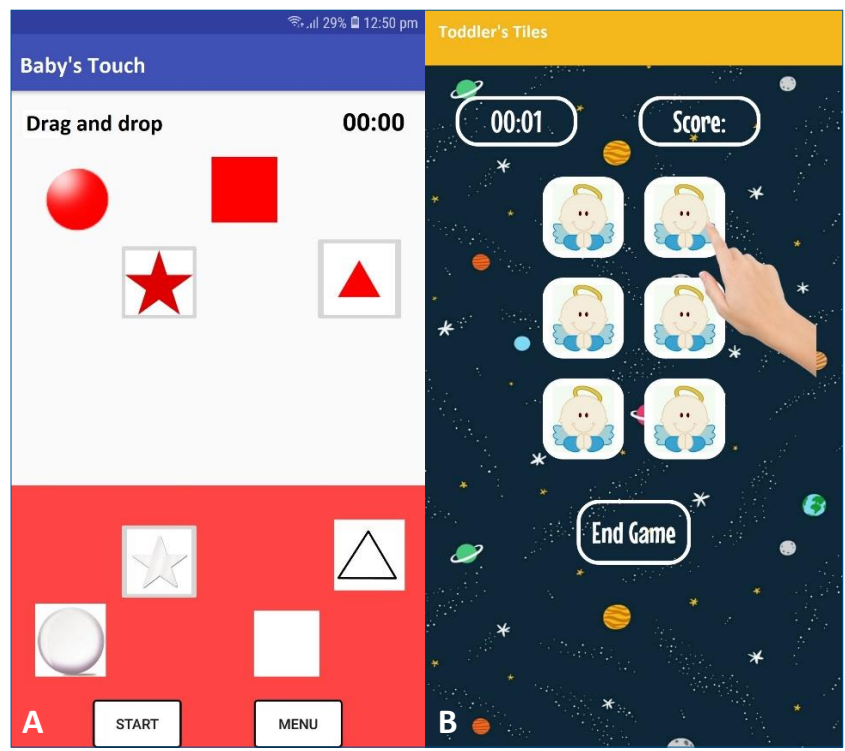

Fig. 1. The 2 apps used in the study. Here, (A) Baby's Touch is being used to study children's ability to perform dragging and dropping maneuvers, and (B) Toddler's Tiles is being used with the animation prompting technique.

techniques (Fig. 1B). The app is in the form of a game. The app can provide instructions on how to play the game using several prompting techniques. The app displays 6 tiles each of which is hiding a fruit. Tapping on a tile reveals the fruit it is hiding. A child can score a point by tapping on 2 tiles hiding the same fruit in consecutive moves. The time taken by a child to understand the instructions and then discover two fruits is noted. If a child is able to discover 2 fruits within a predefined time limit, then we say that the child is able follow that prompting technique successfully. We say that the child has failed to follow that prompting technique, otherwise. The background of the app has been intentionally made complex to simulate commercially available apps. The app can provide instructions to a user on how to play the game in 5 formats, viz. text, on-the-fly textual prompts, audio, video and animation.

We developed Baby's Touch and Toddler's Tiles for studying the interaction of children with smartphones. Both of these apps 
can be downloaded for free from Google Play.

\section{Study design}

We performed our experiment with 90 children in a kindergarten and a primary school in New Delhi in July 2019. We performed our experiment on children of 3 age groups, viz. 2and 3-year-old children, 4- to 6-year-old children, and 7- and 8 -year-old children. We included 15 female and 15 male children of each age group in our study. The experiment was conducted with the permission of the principals of the kindergarten and the primary school.

We asked the children to perform the different touchscreen gestures using Baby's Touch. Each child was given 2 minutes to perform a particular gesture on a set of 4 objects. We noted which gestures the children could perform within the stipulated time.

We then asked the children to use Toddler's Tiles. We arranged the prompting techniques in a decreasing order of their level of difficulty: instructions in textual format, on-the-fly textual prompts, instructions read out loud, video demonstration and demonstration using an animated hand symbol. The children had to understand the instructions provided through the prompting techniques and play the game. We started with the textual prompting technique. Each child was given 2 minutes to discover at least 2 fruits hidden behind the tiles. If a child was successful, then we assumed that the child was able to follow the prompting technique. Otherwise, we provided the next easier prompting technique to the child. If a child was not able to follow any of the 5 in-app prompting techniques, then an observer gave an inperson demonstration on how to use the app to that child. We noted the most difficult prompting technique each child was able to follow and assumed that the child will be able to follow the easier prompting techniques.

We did not apply for, or receive, any approval from any board or committee for this research because of the following reasons. First, this was a techno-behavioral general pediatric study. The study was nonmedicinal, nonintrusive and nonclinical in nature. Second, all the authors are affiliated to a technology university. There is no department or study program related to human subjects in the university. Consequently, there is no internal committee related to research on human subjects in the university.

\section{Data analysis}

We studied the effects of age and gender of the children on their ability to perform touchscreen gestures and follow prompting techniques. We used 1-way analysis of variance to evaluate the relationship between the age of the children and the number of touchscreen gestures they could perform and the number of prompting techniques they could follow. We used independent samples t-test to evaluate the relationship between the gender of the children and the number of touchscreen gestures they could perform and the number of prompting techniques they could follow. All analyses were performed using IBM SPSS ver. 26.0 (IBM Corp., Armonk, NY, USA) at the 5\% significance level.

\section{Results}

Children aged 2 and 3 years could hardly perform any touchscreen gesture (Fig. 2). Only 27\% children in this age group could tap on an intended place on the touchscreen. Alternatively, children aged 4 to 6 years were more proficient in using smartphones. A majority of children in this age group were able

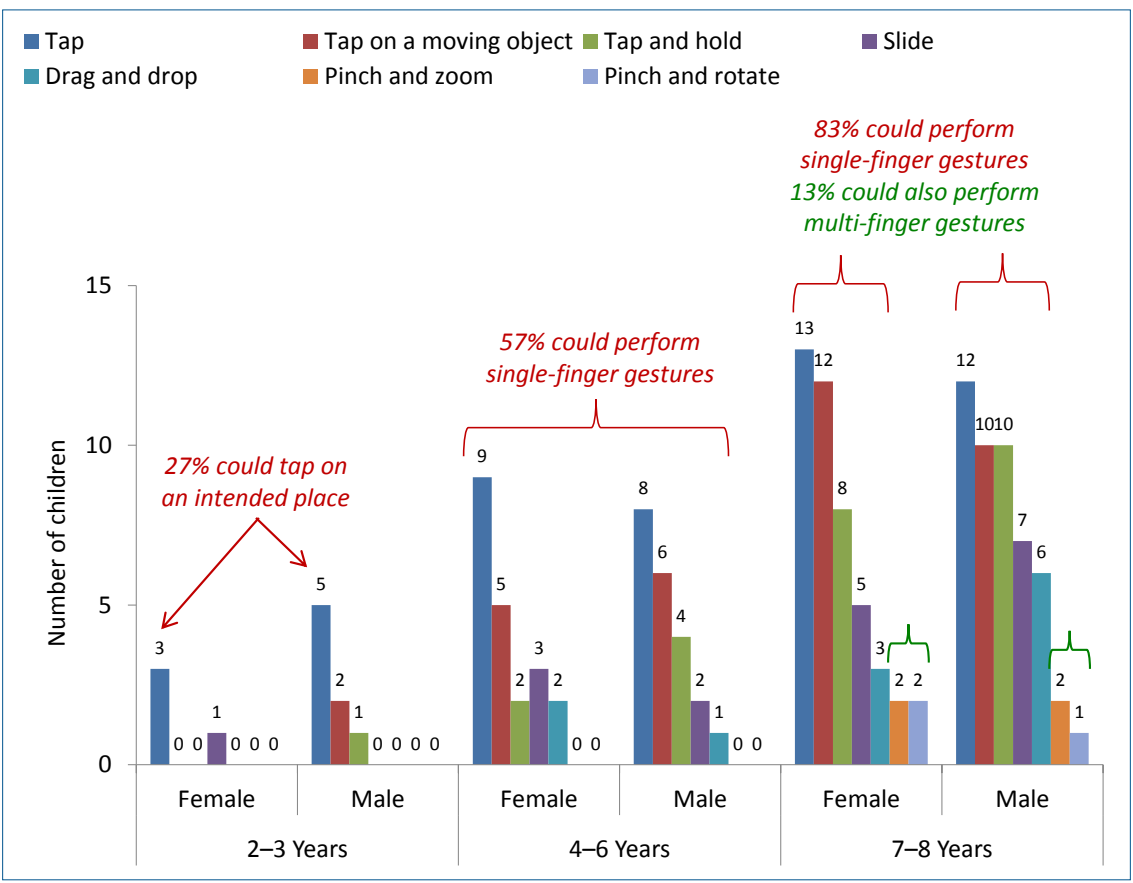

Fig. 2. Touchscreen gestures performed by children of different age groups. Each age group was represented in the study by 15 female and 15 male children. 
to perform simple touchscreen gestures. Among the children in this age group, 57\% could tap on an intended place on the touchscreen, $37 \%$ could tap on an object moving across the screen and 20\% could tap and hold at an intended place on the touchscreen. It was observed that most children learn to perform single-finger touchscreen gestures by the age of 7 years. Children aged 7 and 8 years could perform tap (83\%), tap on a moving object (73\%), tap and hold (60\%), slide (40\%), and drag and drop (30\%). However, only a few children in this age group could perform 2-finger touchscreen gestures like pinch and zoom (13\%) and pinch and rotate (10\%).

Children aged 2 and 3 years could not typically follow any in-app prompting technique (Fig. 3). They can use an app only if someone explained it to them in person. However, children typically develop skills to follow in-app prompting techniques by the age of 4 years. A majority of children aged 4 to 6 years (63\%) could follow one or more in-app prompting techniques. The most difficult prompting technique that children in this age group could follow was instructions in audio format (17\%), instructions in video format (17\%) and instructions using animation (37\%). Almost all children (97\%) aged 7 and 8 years could follow one or more in-app prompting techniques. The most difficult prompting technique that the children in this age group were able to follow was textual instructions (17\%), textual instructions on-the-fly (17\%), instructions in audio format (20\%), instructions in video format (33\%) and instructions using animation (10\%).

We observed a significant difference between the number of touchscreen gestures that the children could perform and the number of prompting techniques that they could follow ( $F=$ $544.0407, P<0.05)$. However, we did not observe a significant $(P>0.05)$ difference in the number of touchscreen gestures that could be performed by female and male children in any of the 3 age groups (Table 1). Similarly, we did not observe a significant $(P>0.05)$ difference in the number of prompting techniques that could be followed by female and male children in any of the 3 age groups (Table 1).

\section{Discussion}

Nowadays, parents and teachers often use smartphones to entertain and educate young children. ${ }^{15)}$ However, smartphones can facilitate child development only if the apps are designed suitably. ${ }^{7)}$ Moreover, children should use mobile apps in conjunction with their academic activities and play in the physical world.

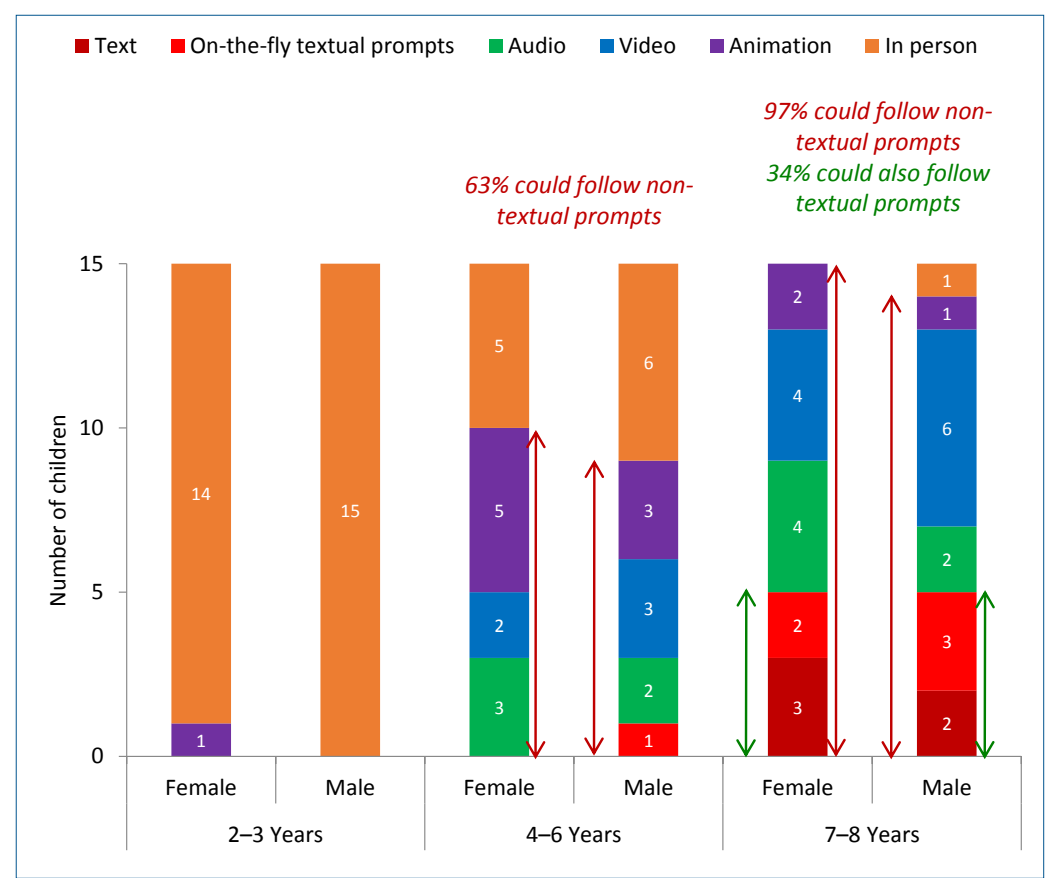

Fig. 3. Prompting techniques that children of different age groups can follow. The most difficult prompting technique that a child was able to follow is shown here. It is assumed that the child will be able to follow the easier prompting techniques.

Table 1. Comparison of performance of female and male children

\begin{tabular}{|c|c|c|c|c|c|c|c|c|}
\hline \multirow{2}{*}{ Age group (yr) } & \multicolumn{4}{|c|}{ Average number of touchscreen gestures performed by children } & \multicolumn{4}{|c|}{ Average number of prompting techniques followed by children } \\
\hline & Female & Male & $t$ value & $P$ value ${ }^{a)}$ & Female & Male & $t$ value & $P$ value $^{\mathrm{a})}$ \\
\hline $2-3$ & 0.33 & 0.73 & -1.356 & 0.186 & 0.07 & 0.00 & 1 & 0.326 \\
\hline 4-6 & 1.40 & 1.40 & 0 & 1 & 1.20 & 1.40 & -0.447 & 0.658 \\
\hline $7-8$ & 2.87 & 2.87 & 0 & 1 & 3.00 & 2.73 & 0.521 & 0.606 \\
\hline
\end{tabular}

${ }^{\mathrm{a}}$ Insignificant differences between female and male children $(P>0.05)$. 
Abdul Aziz et al. ${ }^{10)}$ and Hiniker et al. ${ }^{13)}$ studied the ability of children to use mobile apps and a foreknowledge of their findings helped us to design our study. We designed our experiment to be more systematic and involved more children. We observed that children learn to perform various touchscreen gestures and follow in-app prompting techniques between 4 and 8 years of age. Two- and 3-year-old children typically require help from their caregivers for using mobile apps. Apps for children in this age group should work with little intervention from the children and may ignore accidental touches on the screen. Four- to 6-year-old children can use mobile apps independently. However, using apps meant for children in this age group should require only simple touchscreen gestures like tap and slide. Instructions may be provided through animation which can be understood by a majority of children in this age group. More sophisticated mobile apps may be developed for 7- to 8-year-old children. Sevenand 8-year-old children can perform single-finger touchscreen gestures and follow instructions provided in the forms of animation, and audio and video clips. We observed that some children could perform more touchscreen gestures and follow more prompting techniques than other children of their age. We found that children who use mobile apps frequently could perform more touchscreen gestures and follow more sophisticated prompting techniques. The ability to perform touchscreen gestures or follow prompting techniques is not an appropriate measure of children's development.

Smartphones may be used to foster child development. How. ever, the apps to be provided to children should be developed according to their physical and cognitive capabilities. Children learn to perform single-finger touchscreen gestures and follow nontextual prompting techniques between 4 and 8 years of age. Apps for children aged 8 years or less should not use textual prompts and using such apps should not require performing multifinger gestures like pinch and zoom and pinch and rotate.

\section{Conflicts of interest}

No potential conflict of interest relevant to this article was reported.

\section{References}

1. Eisen S, Lillard AS. Young children's thinking about touchscreens versus other media in the US. J Child Media 2017;11:167-79.

2. Lim CH, Kim EJ, Kim JH, Lee JS, Lee Y, Park SH. The correlation of depression with Internet use and body image in Korean adolescents. Korean J Pediatr 2017;60:17-23.

3. Yadav S, Chakraborty P. Children aged two to four are able to scribble and draw using a smartphone app. Acta Paediatr 2017;106:991-4.

4. Yadav S, Chakraborty P. Using smartphones with suitable apps can be safe and even useful if they are not misused or overused. Acta Paediatr 2018;107:384-7.

5. Hirsh-Pasek K, Zosh JM, Golinkoff RM, Gray JH, Robb MB, Kaufman J. Putting education in "educational" apps: lessons from the science of learning. Psychol Sci Public Interest 2015;16:3-34.

6. Gallup JL, Ray BB, Bennett CA. Leveraging mobile technologies to support active learning for all students: smartphones to support learning. In: Baruch AF, Tal HM, editors. Mobile technologies in educational organizations. Hershey (PA): IGI Global, 2019:302-26.

7. Chau CL. Positive technological development for young children in the context of children's mobile apps [dissertation]. Medford (MA): Tufts University, 2014.

8. Anthony L, Brown Q, Nias J, Tate B, Mohan, S. Interaction and recognition challenges in interpreting children's touch and gesture input on mobile devices. In: Proceedings of the ACM International Conference on Interactive Tabletops and Surfaces; 2012 Nov; Cambridge, MA. New York (NY): Association for Computing Machinery, 2012:225-34.

9. Callaghan MN, Reich SM. Are educational preschool apps designed to teach? An analysis of the app market. Learn Media Tech 2018;43:280-93.

10. Abdul Aziz NA. Children's interaction with tablet applications: gestures and interface design. Int J Comput Inf Tech 2013;2:447-50.

11. Barendregt W, Bekker MM. Children may expect drag-and-drop instead of point-and-click. In: CHI Conference on Human Factors in Computing Systems; 2011 May; Vancouver, BC. New York (NY): Association for Computing Machinery, 2011:1297-302.

12. Nacher V, Jaen J, Navarro E, Catala A, Gonzalez P. Multi-touch gestures for pre-kindergarten children. Int J Hum Comput Stud 2015;73:37-51.

13. Hiniker A, Sobel K, Hong SR, Suh H, Irish I, Kim D, Kientz JA. Touchscreen prompts for preschoolers: Designing developmentally appropriate techniques for teaching young children to perform gestures. In: Proceedings of the Fourteenth International Conference on Interaction Design and Children; 2015 Jun; Boston, MA. New York (NY): Association for Computing Machinery, 2015:109-18.

14. McKnight L, Fitton D. Touchscreen technology for children: giving the right instructions and getting the right responses. In: Proceedings of the Ninth International Conference on Interaction Design and Children; 2010 Jun; Barcelona, Spain. New York (NY): Association for Computing Machinery, 2010:238-41.

15. Yadav S, Chakraborty P. Smartphone apps can entertain and educate children aged two to six years but should be used with caution. Acta Paediatr 2018;107:1834-5. 